\title{
COMPOUND EFFECT OF EHD AND SURFACE ROUGHNESS IN POOL BOILING AND CHF WITH R-123
}

\author{
S.W. Ahmad ${ }^{1}$, T.G. Karayiannis ${ }^{1}$, D.B.R. Kenning ${ }^{1}$ and A. Luke ${ }^{2}$ \\ 1 School of Engineering and Design, Brunel University, Uxbridge, West London, UB3 8PH, UK. \\ 2 Technical Thermodynamics Universitat Kassel Kurt-Wolters-Str.3, 34125 Kassel, Germany.
}

\begin{abstract}
Saturated pool boiling of R-123 at 1 bar, including the critical heat flux (CHF), was enhanced by modifying the surface characteristics and applying a high intensity electrostatic field, the latter termed electrohydrodynamic (and abbreviated EHD) enhancement. The heat flux was varied from very low values in the natural convection regime up to CHF. Experiments were performed with increasing and decreasing heat flux to study boiling hysteresis without and with EHD. Boiling occurred on the sand blasted surface of a cylindrical copper block with embedded electrical heating elements, with standardized surface parameter $\mathrm{P}_{\mathrm{a}}=3.5 \mu \mathrm{m}$. The electric field was generated by a potential of $5 \mathrm{kV}$ to $25 \mathrm{kV}$, applied through a $40 \mathrm{~mm}$ diameter circular electrode of ss-304 wire mesh, aperture size $5.1 \mathrm{~mm}$, located at distances of $5-60 \mathrm{~mm}$ from the surface, with most of the data obtained for $20 \mathrm{~mm}$. The data for the rough surface were compared with earlier data for a smooth surface and indicated a significant increase in the heat transfer rates. EHD produced a further increase in the heat transfer rates, particularly at low heat flux values and near the CHF. Boiling hysteresis was reduced progressively by EHD and eliminated at high field strength.
\end{abstract}

Keywords: pool boiling, surface roughness, critical heat flux, electrohydrodynamic enhancement

\subsection{Introduction}

\section{Surface characteristics}

Various efforts were made in the past to enhance pool boiling heat transfer by applying different passive and active techniques such as the application of high intensity electric fields and changes in surface finish. Nucleate pool boiling at low to moderate heat flux and critical heat flux were examined using different heating surface topographies combined with different geometries: tube and horizontal flat surface. It was also observed that the degree of enhancement differs from fluid to fluid and depends on the material of the surface. Surface topography is mostly characterized by average surface roughness, $\mathrm{R}_{\mathrm{a}}$ or root mean square of the average roughness, $\mathrm{R}_{\mathrm{q}}$. Physical surface enhancement or roughness can be produced by applying sand blasting, emery paper treatment or chemical etching $[1,2]$.

The important reality is that cavities on the heating surface serve as nucleation sites. They are responsible for initiating bubble formation at low superheat. With the increase in the number of nucleation sites, the heat flux at a particular value of wall superheat is increased. Moreover, physical surface treatment increases the average value of surface roughness which increases the nucleation site density [2]. Das and Das [3] examined topographically different surfaces by drilling holes of $600 \mu \mathrm{m}$ in diameter and $2 \mathrm{~mm}$ in depth using distilled water as the working fluid and reported enhancement by upto $100 \%$ when comparing their results with the values for a plain surface. Based on the results from the microdrilled surface Das and Das [3] modified the Yamgate's correlation [cited in reference 3] and proposed the following equation in which the heat flux is a function of nucleation site density and wall superheat.

$q=a(\Delta T)^{b}\left(\frac{N}{A}\right)^{c}$

where $a=7.7655, b=2.0307$ and $c=0.523141$ are empirical constants calculated for distilled water.

A similar increasing trend was observed by Benjamin and Balakrishnan [4] during experiments, which involved boiling at low to moderate heat flux of four different liquids namely: water, acetone, carbon tetrachloride and n-hexane on an aluminum surface $\left(\mathrm{R}_{\mathrm{a}}=1.17 \mu \mathrm{m}\right)$. The heat flux increased by up to $25 \%$ in water, $90 \%$ in carbon tetrachloride, $122 \%$ in acetone and $60 \%$ in n-hexane compared to a similar experiment on an aluminum surface of $\mathrm{R}_{\mathrm{a}}=0.089 \mu \mathrm{m}$ at a specific value of superheat. Similar to the above results, the experiments with refrigerants R-134a and R-407c reported by Hsieh and Weng [5] revealed that as the surface roughness was increased pool boiling was enhanced. They used $19 \mathrm{~mm}$ (OD) copper tubes and used emery paper \# 20, 50, 100 and 150 to treat the surface. The surface roughness $R_{a}$ was reported as 4.57, 3.95, 2.12 and $1.82 \mu \mathrm{m}$ respectively. They gave no further details on how they actually used the paper on the tubes (e.g. pressure applied and direction). The enhancement in the heat transfer coefficient ratio of the emery treated tubes over a polished tube (surface roughness $0.03 \mu \mathrm{m}$ ) was reported to be upto 2.36 for R-134a and 1.55 for R-407c at the heat flux value of $10 \mathrm{~kW} / \mathrm{m}^{2}$. Kang [6] observed a $71.4 \%$ rise in pool boiling heat flux of water when there was an increase of $300 \%$ in surface roughness with horizontal tubes.

Gorenflo et al. [7] reported that the surface treatment methods play a vital role in the heat transfer rates during pool boiling. They performed experiments on horizontal copper tubes $(8$ $\mathrm{mm}$ OD). The outer surfaces of the tubes were treated in three different ways namely: fine sandblasting, fine + medium sandblasting and emery grinding. During the fine sandblasting procedure, corundum grains $\mathrm{F} 320\left(\mathrm{~d}_{\mathrm{p}}=20-30 \mu \mathrm{m}\right)$ were used at 3 bar pressure (the detailed sandblasting procedure is described in reference [8]). For fine + medium sandblasting method, the fine sand blasting was performed as mentioned above and for the medium sandblasting corundum grains $\mathrm{C} 220\left(\mathrm{~d}_{\mathrm{p}}=50-80 \mu \mathrm{m}\right)$ were utilized at 1.5 bar pressure. The third tube was grinded with emery paper grade \# 400. The surfaces were characterized by standardized surface parameter $\mathrm{P}_{\mathrm{a}}$ (according to DIN EN ISO 4287) and reported as $0.58 \mu \mathrm{m}$, $0.27 \mu \mathrm{m}$ and $0.56 \mu \mathrm{m}$ for emery grounded, fine sandblasted and fine + medium sandblasted copper tubes respectively. The results obtained from the pool boiling tests of normal propane 
at a saturated pressure of 4.247 bar revealed that at a given value of heat flux the heat transfer coefficient was greater for both sandblasted tubes than the emery treated in the nucleate boiling regime. Luke [9] provided a possible explanation by analyzing the surface roughness profiles of the tubes as shown in figure 1 . The standardized surface parameter $\mathrm{P}_{\mathrm{a}}$ is almost the same for both sandblasted and emery treated tubes. From the overview analysis of surface profile, it can be observed clearly that on the emery ground surface there were deeper valleys and higher peaks than the sandblasted surface. On the other hand, there was a more homogenous cavity distribution on the sandblasted surface $[9,10]$. Furthermore, the surface microstructure was explained by Luke [11] analysing the surface roughness profile using a ball rolling procedure $\left(\mathrm{R}_{\mathrm{B}}=\right.$ 25, 250 and $2500 \mu \mathrm{m}$ discussed by Gorenflo et al. [7] in detail) and statistical techniques and found that the density of cavities was higher on the sandblasted surface than on the emery grounded surface. Moreover, the analysis of fine sandblasted and fine + medium sandblasted revealed that the cavities on the fine + medium sandblasted surface were bigger than on the fine sandblasted surface [11]. Similar to these observations, Kotthof et al. [12] analysed a new modified surface which was prepared by fine + medium sandblasting and then was rolled to create re-entrant cavities. After detailed surface profile and statistical analysis, it was reported by Luke [11] and Kotthof et al. [12] that before rolling there were sharp edges in the bottom of the cavities and the surface between two cavities. But after the rolling process the surface in the bottom and between the cavities flattened and the mouth of the cavity became narrow. The vapour could be trapped more effectively in narrow mouth cavities which resulted in augmentation in heat transfer rates. From the above discussion it can be concluded that the method of surface preparation should be considered and careful surface finish may lead to more effective and smaller equipment design for given thermal loads.
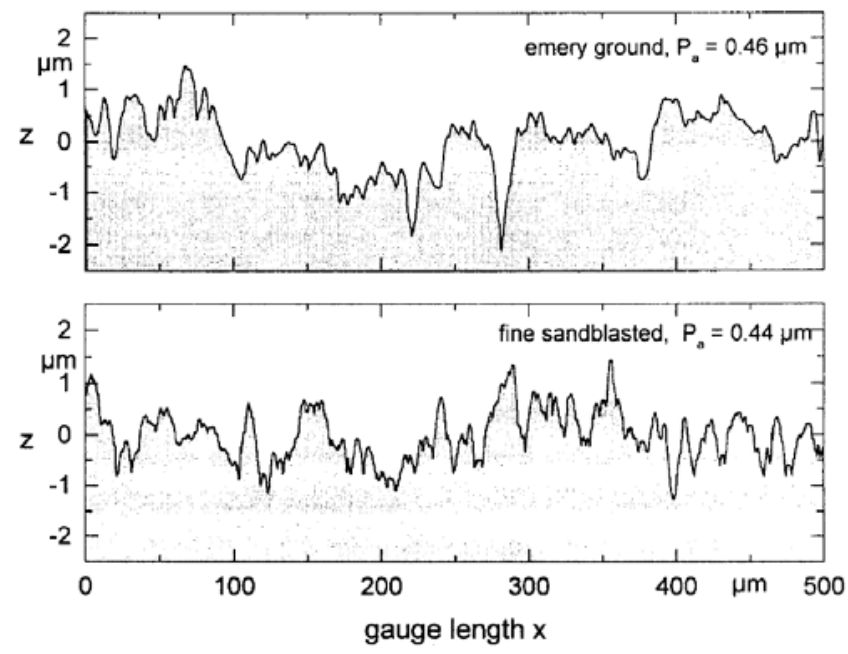

Figure 1 Roughness profile of emery grounded and fine sandblasted copper surfaces [9].

The influence of surface characteristics on the critical heat flux was examined by Ferncic and Golobic [13] in experiments with saturated boiling of water at atmospheric pressure on steel. Topographically different surfaces of steel 1010 were investigated by treating the surface with sand paper of different grade numbers, i.e. $600,400,320,150,80$ and 50 to obtain the average roughness $\mathrm{R}_{\mathrm{a}}=0.1,0.2,0.3,0.6,1.0$ and $1.5 \mu \mathrm{m}$ respectively. The results were presented in the form of the equation below:

$\mathrm{q}_{\mathrm{CHF}}=\mathrm{a} \ln \mathrm{R}_{\mathrm{a}}+\mathrm{b}$

where $\mathrm{a}$ and $\mathrm{b}$ are empirical constants dependent on the properties of the boiling liquid and heating surface. From this work it can be concluded that the $\mathrm{q}_{\mathrm{CHF}}$ increases with increasing the surface roughness. Theofanous [14] also observed an increase in the critical heat flux with an increase in nucleation site density.

\section{Electrohydrodynamics}

The importance of the EHD technique for the augmentation of boiling heat transfer has been reported by numerous researchers. Particularly, the significant increase in the heat transfer coefficient was reported in nucleate low heat flux and critical heat transfer regions in pool boiling. Allen and Karayiannis [15] produced a review of the EHD work up to 1994. Yabe et al. [16] presented a further review type chapter in 1999. The body force which is the result of the application of the high intensity electric field is given by

$$
\bar{f}_{e}=\rho_{e} \bar{E}-\frac{1}{2} E^{2} \nabla \varepsilon+\frac{1}{2} \nabla\left[E^{2} \rho\left(\frac{\partial \varepsilon}{\partial \rho}\right)_{T}\right]
$$

The symbols are defined in the nomenclature. The first term on the right hand side of equation (3) is the electrophoretic force acting on free charges in the medium. The second term is due to spatial changes in the electrical permittivity of the medium. The third term represents the dielectrophoretic and electrostrictive forces within the fluid, Pohl [17]. The local electric field strength $(E=-\nabla V)$ depends on the geometries of the heated surface and the electrodes, the presence of bubbles and the local values of the electric permittivity and conductivity, Hristov et al. [18] Allen and Karayiannis [15] and Karayiannis and $\mathrm{Xu}$ [19].

A report on the enhancement of pool boiling heat transfer under the influence of EHD was offered by Yokoyama et al. [20]. They performed an experiment under EHD pool boiling conditions using R-11 as the working fluid where the test surface was a smooth copper plate. At very low heat flux, about $2.8 \mathrm{~kW} / \mathrm{m}^{2}$, there was no boiling from the heating surface but tiny bubbles were observed from the edges of the surface. As the heat flux increased, but still at low values and in the absence of an electric field, they noted that the bubble production from the heating surface was uniform. When the electric field was applied the bubble generation was suppressed. This bubble suppression effect increased as the applied voltage was increased to $26 \mathrm{kV}$. However, at high heat flux values the bubble generation became more vigorous upon the application of EHD. The rise in the heat transfer coefficient at low heat flux was hypothetically explained by Zaghdoudi and Lallemand [21] as follows: at low heat flux, natural convection is responsible for heat transfer. A thermal boundary layer forms over the heating surface while the bulk fluid is under saturation temperature at a specific pressure. When a high intensity electric field is applied, dielectrophoretic and electrostrictive forces appear within the thermal boundary layer, i.e. due to the thermal gradient within the boundary layer, electric conductivity and permittivity vary and this produces dielectrophoretic and electrostrictive forces. Note that these forces are negligible within the bulk fluid due 
to the uniform temperature. According to Zaghdoudi and Lallemand [21] this difference in the forces gives rise to electroconductive movement and the thickness of the thermal boundary decreases; and hence the wall temperature also decreases. Of course one could argue that this drop in the wall temperature (wall superheat) results in the suppression of boiling while at the same time it provides improved heat transfer rates.

Baboi and Bologa [22] suggested that bubbles growing from vapour nuclei coalescence with other bubbles and form larger bubble detachment diameters. According to [22], the electric field acts on these bubble - generating nuclei and tends to make them inactive. As a result, at constant heat flux the bubble departure diameter decreases. Furthermore, with increasing electric field strength at constant heat flux bubbles almost disappear agreeing with the observation of [20].

\section{Effect of Fluid properties}

The effect of fluid properties under the influence of EHD was examined by Zaghdoudi and Lallemand [21]. They performed pool boiling experiments under saturated atmospheric conditions using R-123, R-113 and n-pentane on a copper surface polished with emery paper $\mathrm{N}$ 600. The electric field was provided by using a $2 \mathrm{~mm}$ mesh as one electrode while grounding the heating surface, which serves as the second electrode. The electric potential was varied from $0-25 \mathrm{kV}$. They observed that the heat transfer coefficient increased by $160 \%, 170 \%$ and $600 \%$ for $\mathrm{n}$ - pentane, R113 and R-123 respectively. The values of charge relaxation time of $\mathrm{n}$ pentane, R-113 and R-123 are $3.40 \times 10^{-3} \mathrm{~s}, 2.76 \times 10^{-3} \mathrm{~s}$ and $0.9 \times 10^{-3} \mathrm{~s}$ respectively [21]. They concluded that the EHD enhancement is dependent on the relaxation time, i.e. the smaller the charge relaxation time the greater the heat transfer enhancement under the influence of EHD. Earlier Karayiannis [23] reported tests with R-11 and R-123 in a five-tube shell and tube heat exchanger. The high intensity electric potential was provided by using fourteen mild steel rods and was varied from $0-25 \mathrm{kV}$. They noticed that, in case of R-123, augmentation using EHD was significant especially at low heat flux values and was about 9.3 at $5 \mathrm{~kW} / \mathrm{m}^{2}$. In case of R11 , the augmentation was marginal which indicates that EHD is strongly dependent upon the properties of the fluid; the charge relaxation time of $\mathrm{R}-123$ and $\mathrm{R}-11$ is estimated to be $0.9 \times 10^{-3} \mathrm{~s}$ and $1.3 \mathrm{~s}$ respectively [reported in reference 18 ]. Furthermore, Ohadi et al. [24] investigated the EHD effect on pool boiling of R-123 and R-11 with oil concentration $(0,2 \%$, and $5 \%$ by weight) on the shell side of a shell and tube heat exchanger. It was observed that R-123 exhibited a high EHD augmentation as compared to R-113 in pure form. The authors stated that this was due to the difference in permittivity of the two fluids, which of course relates to differences in the charge relaxation time. The maximum enhancement was $450 \%$ and $170 \%$ for $\mathrm{R}-123$ and $\mathrm{R}-11$ respectively. It was also noticed that with the oil addition the enhancement factor decreases. Contradicting the above three studies, which noted significant enhancement with R-123 [21,23,24] and smaller or marginal enhancement with R-11, Yokoyama et al. [20] noticed that the heat transfer augmentation at low heat flux with R11 was 3 to 7 times under the influence of EHD at an electric potential of $26 \mathrm{kV}$ on a smooth copper heating surface. However, they did not compare directly (experimentally) with other fluids. It can be concluded from the above review that fluid properties play a significant role in the heat transfer augmentation when high intensity electric field is employed. It can be further stated that it is difficult to measure and control the effect of accidental contamination of water, dissolved gases and the decomposition of the fluid, which results in poor reproducibility and repeatability of experimental data between different laboratories.

\section{Boiling Hysteresis}

Zaghdoudi and Lallemand [21] reported that the hysteresis phenomenon almost disappeared by applying $2000 \mathrm{kV} / \mathrm{m}$. Basu [25] performed an experiment with carbon tetrachloride, using platinum-iridium wire of diameter $0.04 \mathrm{~mm}$ and both $\mathrm{AC}$ and DC electric fields. Hysteresis was removed more effectively with the application of DC electric field than with $\mathrm{AC}(500 \mathrm{kV} / \mathrm{m})$. Also good augmentation at the low heat flux region was reported. Cooper [26] performed a pool boiling experiment on R-114 using a low-fin tube surface. With EHD, enhancement was noted in all the boiling regimes. It was also observed that at low heat flux hysteresis was eliminated. It was proposed that the EHD is responsible for activation of nucleation sites which remain active even if EHD is removed. This seems to contradict the finding of [20] and [22] above. However, the value of the electric field applied, the fluid involved and the actual value of the heat flux may lead to a different response of the boiling fluid to EHD.

\section{Effect of EHD on the critical heat flux}

Berghmans [27] mathematically examined the effect of EHD on $\mathrm{CHF}$ and suggested that vapour columns formed on the heating surface near the critical heat flux are destabilized by applying DC electric field. This destabilization is responsible for the increase in the CHF. He derived a mathematical expression to predict maximum heat flux based on the hydrodynamic theory presented by Zuber [28] as follows:

$q_{\max }=\frac{1}{8}\left(\frac{\pi}{3}\right)\left(\varepsilon_{g} \rho_{g}\right)^{\frac{1}{2}} \frac{\Delta V}{d} h_{\mathrm{lg}}$

Equation (4) was found to be in good agreement with the experimental work of Markel and Durfee [29] who used isopropyl alcohol and distilled water as working fluids. Earlier Johnson [30] derived an expression to estimate the CHF under the influence of the electric field. He considered the electric field effect on hydrodynamic stability and the analysis resulted to the following expression:

$$
\begin{aligned}
& \frac{\left(q_{c}\right)_{E}}{\left(q_{c}\right)_{0}}=\left[\frac{f_{1}\left[1+\sqrt{1+\frac{3\left(\rho_{l}-\rho_{g}\right) g \sigma}{f_{1}^{2}}}\right]}{\sqrt{3\left(\rho_{l}-\rho_{g}\right) g \sigma}}\right] \\
& f_{1}=\left(\frac{\varepsilon_{g}}{\varepsilon_{l}}\right) \frac{\left[\varepsilon_{l}-\varepsilon_{g}\right]^{2}}{\varepsilon_{l}+\varepsilon_{g}} E^{2}
\end{aligned}
$$

Lovenguth and Hanesian [31] noted that the critical heat flux increases with the increase in the DC non - uniform electric field using four different dielectric fluids namely: Freon 113, Carbon tetrachloride, Chloroform and Freon 21. 
Furthermore, they derived an expression based on the Kelvin - Helmholtz instability to predict the effect of DC non uniform electric field on the critical heat flux, as follows [31]:

$$
\begin{aligned}
& \left(\frac{q(E)}{q(0)}\right)_{C H F}=\left[\frac{B E^{2}}{\sqrt{3\left(\rho_{g}-\rho_{v}\right) \sigma g}}+\left[\left[\frac{B E^{2}}{\sqrt{3\left(\rho_{g}-\rho_{v}\right) \sigma g}}\right]^{2}+1\right]^{\frac{1}{2}}\right]^{\frac{1}{2}}(7) \\
& B=\frac{\varepsilon_{g} \varepsilon_{l}\left(\varepsilon_{l}-\varepsilon_{g}\right)^{2}}{\varepsilon_{g}\left(\varepsilon_{l}+\varepsilon_{g}\right)}
\end{aligned}
$$

Baboi and Bologa [22] suggested that at the CHF point the bubble coalescence becomes higher in volume, which covers the entire heating surface. It results in stopping the colder liquid from reaching the surface. Due to this process equipment burnout happens. But with the application of electric field the size of bubble detachment diameter decreases and colder liquid remains available even at higher heat fluxes. This results in increasing the CHF to higher wall superheat with the application of the electric field. Moreover, the hydrodynamic theory does not explain any effect of surface roughness on the CHF, which was reported by Ferncic and Golobic [13] and Theofanous [14]. Further analysis will be therefore required to assess the effect of surface roughness on the CHF.

\section{Summary}

It can be concluded from the above review that the topography of the heating surface can play an enhancing role in pool boiling heat transfer. The structure of the nucleation sites, which can trap the vapour more effectively, is very important for heat transfer augmentation (without EHD). Furthermore, EHD was proved to be an effective active technique for heat transfer enhancement. By the application of high intensity electric field, the dielectric fluid, particularly in the two phase region, becomes polarized and begins to move under the action of EHD forces. These forces are responsible for increasing the effective mixing of the fluid which makes the heat transfer more efficient in the low nucleate boiling region and critical heat flux region, but not so effective in the established boiling regime. The efficiency of the EHD technique is highly dependent on the fluid properties. Moreover, the elimination of hysteresis was also recorded due to the application of EHD. Due to the fact that the effect of EHD is higher near surfaces with fins and protrusions, the compound effect of EHD and surface roughness on pool boiling of R-123 was examined during the present studies. The heating copper surface was sand blasted having a standardized roughness parameter $\mathrm{P}_{\mathrm{a}}=3.5 \mu \mathrm{m}$. The results obtained are compared with past research with the same working fluid.

\subsection{Experimental setup}

The experimental facility comprising a boiling chamber, a condenser, a heating system, a refrigeration system and control and measurement units is shown in figure 2.

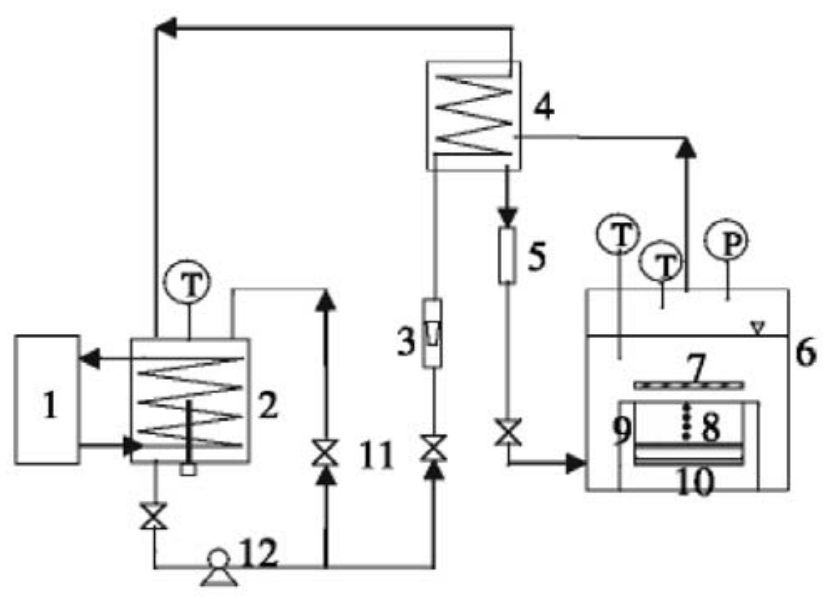

1.R-134a cooling unit 2.Evaporator 3.Water flow meter 4.Condenser 5.Filter/Dryer 6. Boiling chamber 7 .Electrode 8 . Thermocouple 9 . Heating copper block 10 . Heater 11.Valves 12.Pump

Figure 2 Schematic diagram of the experiment facility

The system operated as a thermophyphon in which the vapour of R-123 coming out of the boiling chamber condensed in the heat exchanger mounted above the chamber. The condensate returned back to the boiling chamber via a filter/dryer. The cool water used in the condenser was chilled using an R-134a refrigeration cycle, shown on the left of figure 2.

The boiling chamber was a ss-304 cylindrical vessel $(221 \mathrm{~mm}$ in diameter, $300 \mathrm{~mm}$ in height). Two circular side glasses (100 $\mathrm{mm}$ in diameter) were installed to visually examine the pool boiling process. R-123 was boiled on a sand blasted circular surface (40 $\mathrm{mm}$ in diameter) of a $130 \mathrm{~mm}$ long oxygen free copper block see figure 3 . Six $250 \mathrm{~W}$ cartridge heaters $(6 \mathrm{~mm}$ in diameter, $40 \mathrm{~mm}$ long) were employed to heat the copper block. $\mathrm{K}$ - type thermocouples $0.5 \mathrm{~mm}$ in diameter were placed $2 \mathrm{~mm}, 7 \mathrm{~mm}, 12 \mathrm{~mm}, 17 \mathrm{~mm}, 22 \mathrm{~mm}$ and $27 \mathrm{~mm}$ from the top of the heating surface in holes of $1 \mathrm{~mm}$ at a depth of $10 \mathrm{~mm}$, see figure 3, to record the temperature gradient within the copper block. The power supply to the heaters was controlled by a transformer and measured by a power meter. The boiling surface was prepared at the University of Hannover. The surface was first carefully polished and then sandblasted. The mechanical equipment available for sandblasting included a tube injector which was moved over the surface by a motorized device. Brown aluminum oxide NK 46 (grit size 300-425 $\mu \mathrm{m}$ ) was used as sandblasting material at 3.5 bar pressure while surface to nozzle distance was $60 \mathrm{~mm}$ (the sandblasting procedure is discussed in reference [8]). The surface was characterized by using an ultrasound stylus as explained in reference [11] and the standardized roughness parameter (according to DIN EN ISO 4287) was reported as $P_{a}=3.5 \mu \mathrm{m}$, see figures 4 and 5 . Luke [11] explained the standardized surface parameters $P_{a}$ and $R_{a}$. $\mathrm{P}_{\mathrm{a}}$ is the deviation of the measured 2-D profile ( $=2 \mathrm{D}$ analysis) from the reference ( $=$ ideal) line $\mathrm{z}=0$ which related to the primary profile according to DIN EN ISO 4287, i.e. the profile without cut - off $(\lambda=0)$, whereas $R_{a}$ is the measure of the 2-D deviation with standardized cut - off and in this case filtered profile is used. To avoid any misinterpretation, the effect of filtering the value of the standardized surface roughness is reported in $\mathrm{P}_{\mathrm{a}}$ [discussed in detail in reference11]. A pressure gauge and a pressure transducer were 
connected at the top of the chamber to monitor the pressure. The temperature inside the chamber was monitored using three $\mathrm{K}$ - type thermocouples - two in the liquid and one in the vapour region. The difference between the three thermocouples was less than the calibrated error of the thermocouples $( \pm 0.2 \mathrm{~K})$. The saturation temperature $\left(T_{\text {sat }}\right)$ was recorded from one of the thermocouples located in the liquid region near the heated surface.

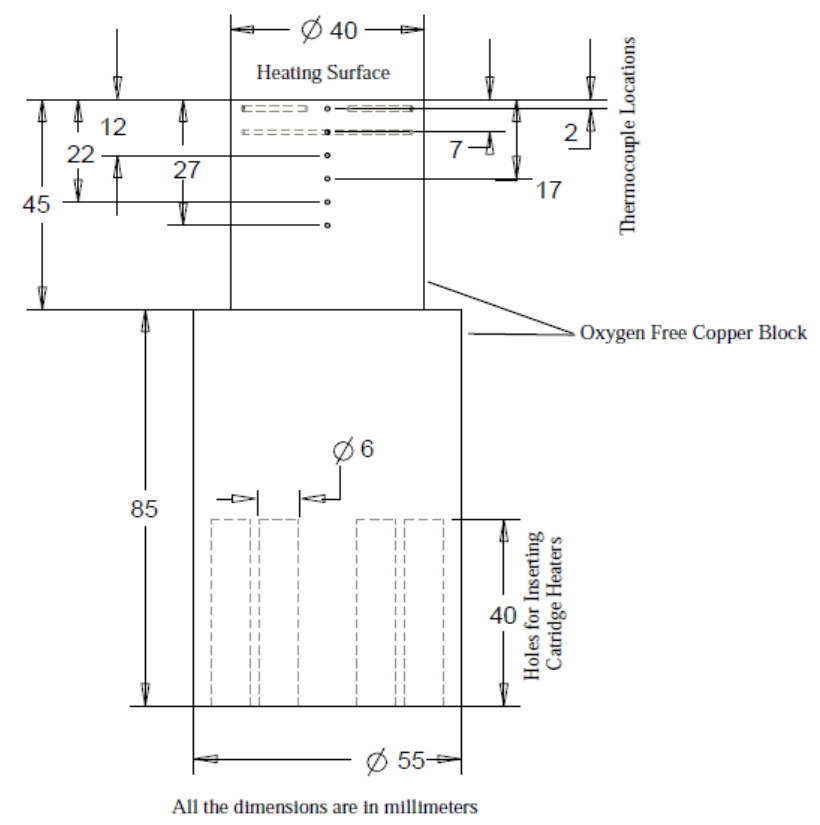

Figure 3 Schematic diagram of the heating surface/block.

A high voltage DC (+ve) power supply (Model AU-30P 3.3 Matsusoda precision Inc) was used to generate the electric potential from 0 to $25 \mathrm{kV}$. A mesh electrode (ss-304, $5.1 \mathrm{~mm}$ aperture, free area fraction 0.64 ) $40 \mathrm{~mm}$ in diameter was connected to the high voltage generation and the heating surface was earthed. The distance between the electrode and the heating surface was varied from $5 \mathrm{~mm}$ to $10,20,40$ and 60 $\mathrm{mm}$, but most of the data were recorded at the $20 \mathrm{~mm}$ electrode spacing. The mesh electrode was sandwiched between two PTFE washers having $40 \mathrm{~mm}$ inner diameter, which were supported by three PTFE rods to isolate the electrode from the rest of the chamber.

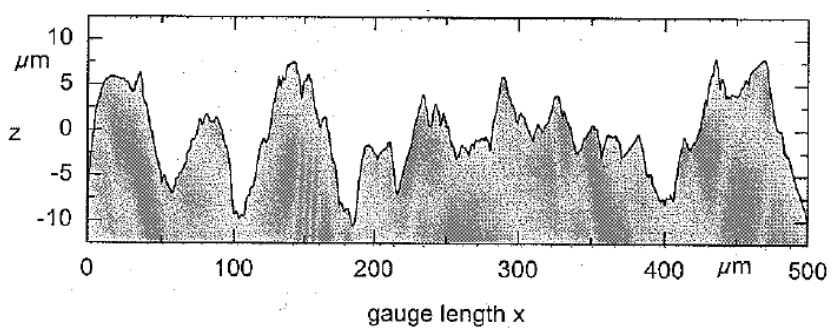

Figure 4 Profile of heating surface (enlargement $\mathrm{z} / \mathrm{x}=$ 6.35), $\mathrm{P}_{\mathrm{a}}=3.5 \mu \mathrm{m}$.

\subsection{Experimental Procedure and Data Analysis}

The boiling chamber and condenser were filled with nitrogen at a high pressure of about 2.5 bar for a leakage test. After the system was made leak-free, the chamber was evacuated and

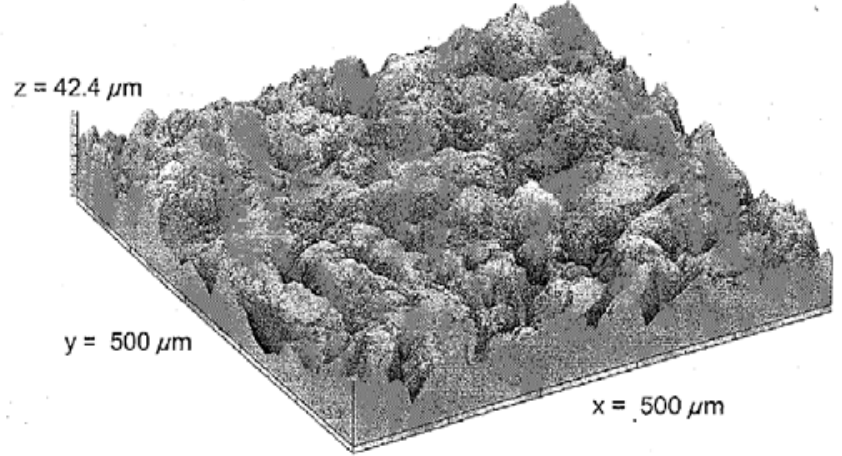

Figure 5 Topography of heating surface (enlargement $\mathrm{z} / \mathrm{x}=$ 1.94), $\mathrm{P}_{\mathrm{a}}=3.5 \mu \mathrm{m}$.

filled with R-123 up to $80 \mathrm{~mm}$ above the heating surface. The fluid was boiled at moderate heat flux for about 30 minutes to remove any noncondensable gases, which were vented from the system through a valve located at the top of the condenser. The system was then sealed and the pressure was controlled by the flow of chilled water in the condenser. The measurements were recorded at saturated conditions $(101 \mathrm{kPa})$ when the system reached steady state, i.e. the pressure remained stable for 10 minutes or temperature was only within $\pm 0.1 \mathrm{~K}$ during this period. Measurements were recorded for both increasing and decreasing heat flux. For CHF measurements, the heat flux was increased further until the temperature within the copper block started increasing rapidly. The readings from the six thermocouples are used to obtain the temperature gradient in the copper block and hence the heat flux as follows

$$
q=k \frac{d T}{d x}
$$

The radial heat flux was observed by placing five thermocouples, $2 \mathrm{~mm}$ from the heating surface, inside the heating block. The angles between the thermocouples were $0^{0}$, $60^{\circ}, 120^{\circ}, 180^{\circ}$ and $270^{\circ}$ to each other. It was observed from the readings of the thermocouples that the radial heat flow was negligible. Therefore, it is assumed that the heat transfer through the copper block is one-directional and the temperature along the radius of the copper block at any point are

constant.

The heat transfer coefficient on the liquid side is then given by

$$
h=\frac{k}{T_{\text {sat }}-T_{w}} \frac{d T}{d x}
$$

where $T_{\text {sat }}$ is the bulk temperature of the fluid and $T_{w}$ is the temperature of heating surface.

All the sensors were calibrated, i.e. the thermocouples were calibrated against a precision thermometer (F 250MKII, Automatic System Laboratories) and the pressure transducer against a dead weight pressure tester. The accuracy in the measurements of the pressure transducer was $\pm 0.5 \mathrm{kPa}$. As mentioned above, the error in the thermocouples was estimated to the $\pm 0.2 \mathrm{~K}$. The error in the location of the thermocouples in the block was estimated to be $\pm 0.05 \mathrm{~mm}$. The propagation of the error was based on the equation (11) below, see reference [32]. The change in the conductivity of 
copper was negligible over the range of copper block temperature).

$\left[\frac{U_{h}}{h}\right]^{2}=\left[\frac{U_{m}}{m}\right]^{2}+\left[\frac{U_{K}}{K}\right]^{2}+\left[\frac{U_{T w}}{T_{w}-T_{\text {sat }}}\right]^{2}+\left[\frac{U_{\text {Tsat }}}{T_{w}-T_{\text {sat }}}\right]^{2}$

It was found that the percentage error in the heat transfer coefficient was 2.5 to $5 \%$ and the error in the heat flux was 2 to $4 \%$.

\subsection{Results and Discussion}

\subsection{Effect of surface roughness (without EHD)}

The first test of the roughened surface involves the examination of the heat transfer characteristics without an electric field. The heat flux as a function of wall superheat is shown in figure 6.

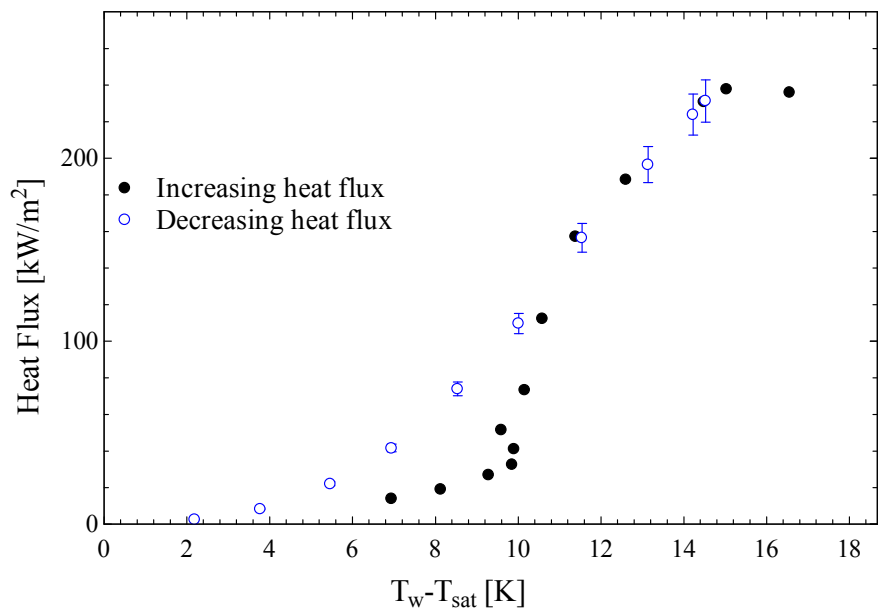

Figure 6 Boiling curves for R-123 with increasing and decreasing heat flux, without EHD.

The results generate the typical boiling curve and demonstrate the difference for increasing and decreasing heat flux, i.e. boiling hysteresis with some cavities not active in case of increasing heat flux and remaining active when activated at higher heat fluxes. The results are compared with the Roshenow correlation equation (12) below [as cited in reference 33], the published data of Zahghdoudi and Lallemand [21] and the data of Hristov et al. [18] obtained with an earlier version of the present apparatus, all for R123 at a pressure of $101 \mathrm{kPa}$.

$\frac{c_{p l} \Delta T}{h_{\mathrm{lg}}}=C_{s f}\left[\frac{q}{h_{\mathrm{lg}} \mu}\left[\frac{\sigma}{g\left(\rho_{l}-\rho_{g}\right)}\right]^{\frac{1}{2}}\right]^{m}\left[\frac{c_{p l} \mu_{l}}{k_{l}}\right]^{n}$

Jabardo and Silva [33] calculated the constants in equation (12) to be $\mathrm{m}=0.33, \mathrm{n}=1.7$ and $\mathrm{C}_{\mathrm{sf}}=0.0027$.

The results are in good agreement with the Rohsenow correlation. Zaghdoudi and Lallemand [21] polished the heating surface using emery paper N 600 while Hristov et al. [18] used emery paper P 1200 . The signs $\mathrm{N}$ and $\mathrm{P}$ were used by the authors. Values of surface roughness were not specified but Shahryari et al. [34] polished samples of stainless steel 316 using emery paper of various grits and reported that as the grit number increased the average surface roughness $\left(\mathrm{R}_{\mathrm{a}}\right)$ of the sample decreased. Therefore, the heating surfaces used by
Zaghdoudi and Lallemad [21] and Hristov et al. [18] would have been significantly smoother than the present one with the Hristov et al. [18] surface being the smoothest. This is consistent with the large shift of their boiling curves to the right.

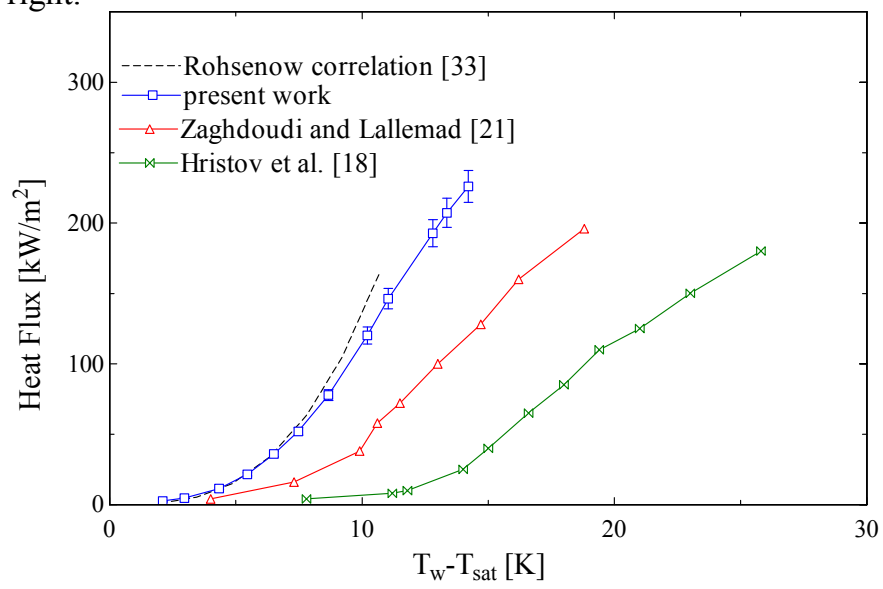

Figure 7 Comparison of present results with past work, without EHD

Figure 8 shows the augmentation ratio (defined as the value of the present heat flux to the value reported in past works at a specific value of superheat) at a given wall superheat compared to [18] and [21]. The augmentation ratio decreases with increasing degree of superheat (heat flux). The slope is higher in comparison with the work of Hristov et al. [18]

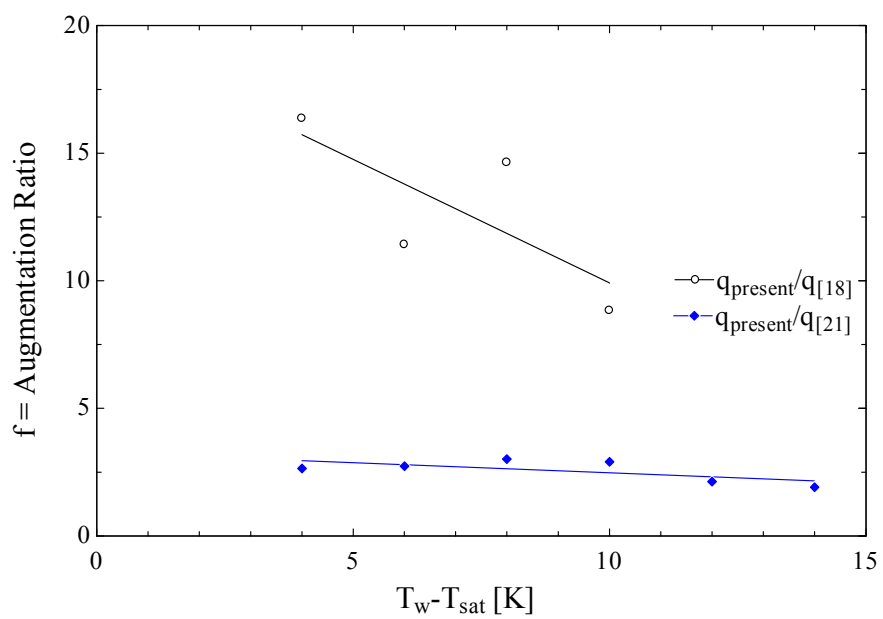

Figure 8 Heat transfer augmentation due to surface roughness, without EHD.

\subsection{Application of EHD}

\subsubsection{With fixed electrode spacing}

For these experiments, the electric field was applied to the mesh electrode placed $20 \mathrm{~mm}$ above the heating surface. The boiling curve hysteresis that is evident in Figure 9 without an electric field is progressively reduced as the electric potential is increased and is eliminated at the highest potential of $20 \mathrm{kV}$, $\mathrm{E}=1000 \mathrm{kV} / \mathrm{m}$. This demonstrates a similar trend to that reported by Basu [25].

According to Copper [26] boiling is strongly dependent on the active nucleation sites on the heating surface which was observed to be activated by the electric field during his experiments. As also reported by Cooper [26], it appears that EHD increases the number of active nucleation sites which could remain active after the field is removed. 


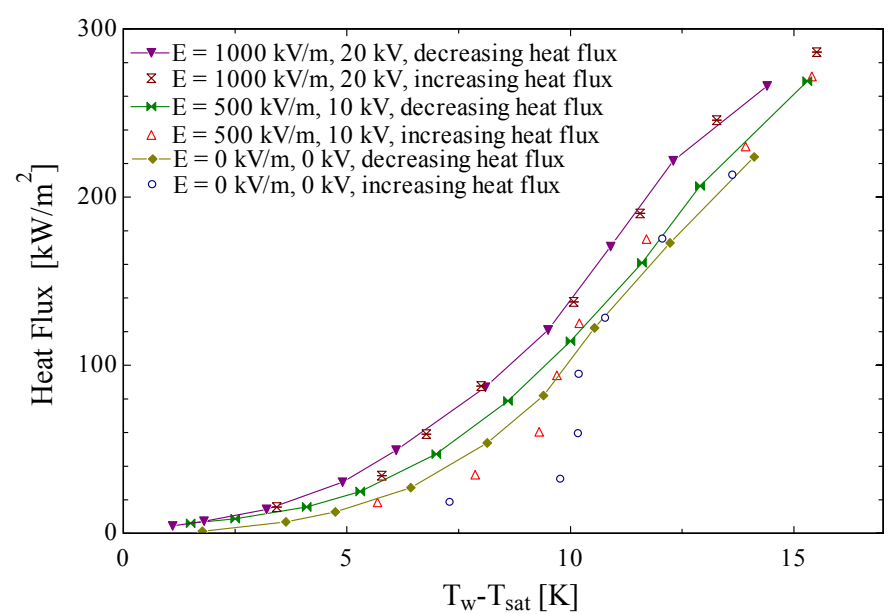

Figure 9 Heat flux versus increasing and decreasing $\Delta \mathrm{T}$

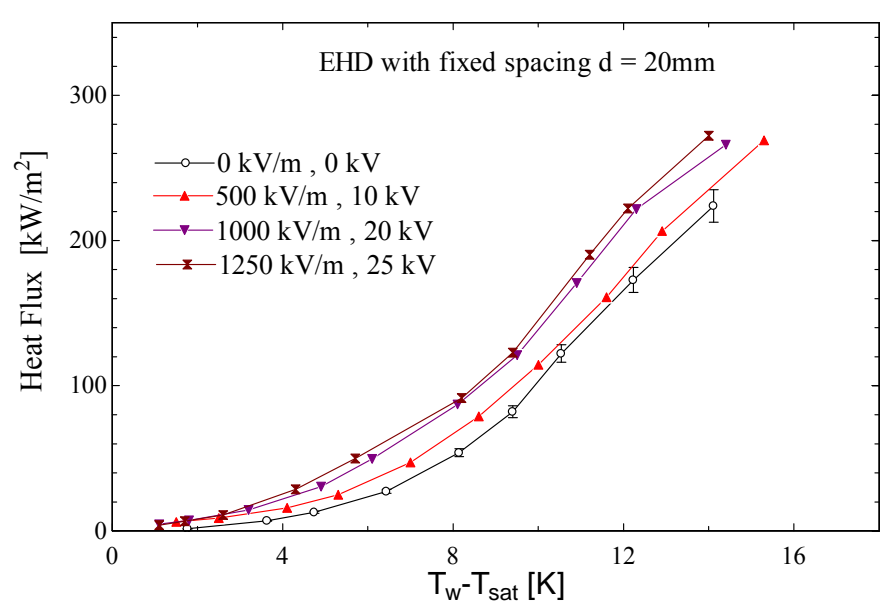

Figure 10 Boiling curves with decreasing heat flux

In Figures 10 and 11 the heat flux and heat transfer coefficient respectively are plotted as functions of the wall superheat showing that the enhancement of heat transfer increases as the potential increases at fixed electrode spacing. As seen in Figure 12, heat transfer enhancement is higher at low heat flux and decreases as the heat flux is increased. It is proposed that two factors are involved in this process:

(i) EHD induced convection occurs at low heat flux, when there were no bubbles seen on the heating surface up to $3 \mathrm{~K}$ wall superheat during the present study.

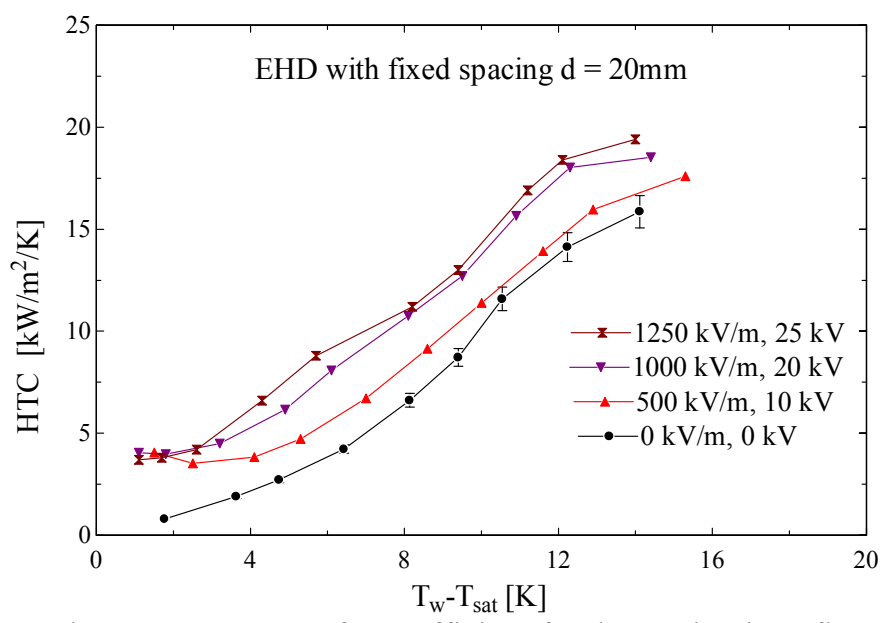

Figure 11 Heat transfer coefficient for decreasing heat flux
As mentioned earlier it is expected that, at this stage, a thin non-homogenous thermal boundary layer covers the boiling surface when there is no electric field applied. The application of EHD affects the thermal boundary layer and increases the circulation rate to bring cold liquid to the heated surface at a faster rate.

(ii) When bubbles appear in the nucleate boiling region electroconvection continues to play a role but is now complemented by the effect of the electric field on the bubbles. Allen and Karayiannis [15] reviewed the EHD effect during two-phase heat transfer and reported that the enhancement could be attributed to the action of electric field on the vapour - liquid interface, vapour bubbles and the possible change in the surface tension and contact angle. The EHD acts to destabilize the vapour - liquid interface and this destabilizing effect tends to reduce the thermal resistance. Furthermore, Karayiannis and $\mathrm{Xu}$ [19] reported that EHD can change the bubble dynamics which may be responsible for heat transfer enhancement. The effect of EHD on the surface tension and contact angle may be responsible for initiation of boiling at low superheats [15]. The combination of the mechanisms may explain the disagreement between observations in references [20, 22, and 26] discussed earlier.

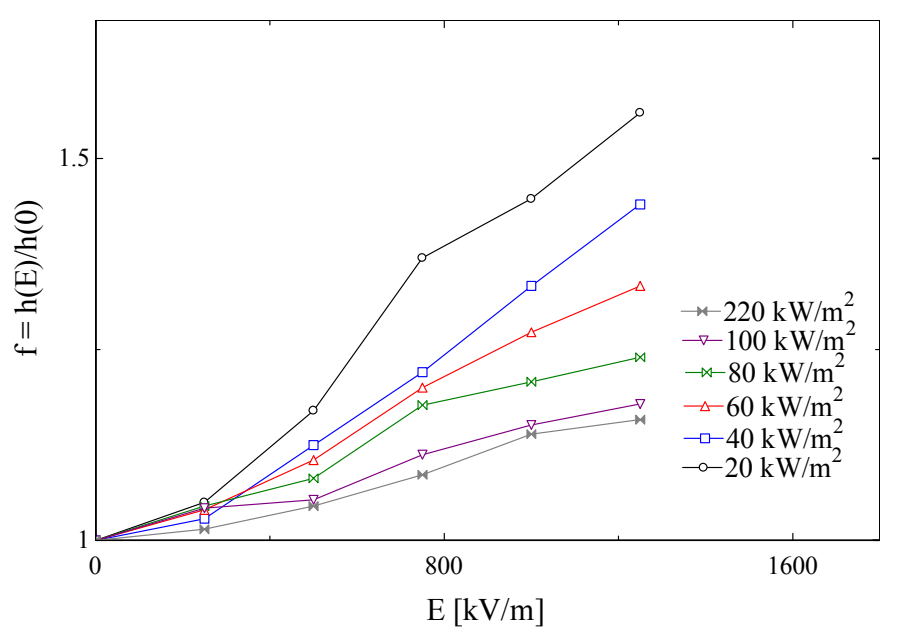

Figure 12 Heat transfer augmentation at fixed electrode spacing, $20 \mathrm{~mm}$.

\subsubsection{Effect of variable electrode spacing (d)}

As shown in the preceding section, the enhancement of heat transfer increases as the electric potential is increased at fixed electrode spacing. The effect of changing the electrode spacing at fixed electric potential is shown in figure 13 for decreasing heat flux. According to Eq. (3), the electrohydrodynamic forces depend on the field strength, the nominal value of which is given by $E=V / d$. Hence, as expected, the highest enhancement, at constant voltage, is obtained with the electrode closest to the heating surface (highest $\mathrm{E}$ ). Two combinations of $\mathrm{V}$ and $\mathrm{d}$ that give the same field strength $\mathrm{E}=500 \mathrm{kV} / \mathrm{m}$ are compared in figure 14 for 20 $\mathrm{mm}, 10 \mathrm{kV}$ and $40 \mathrm{~mm}, 20 \mathrm{kV}$. The effectiveness of the electric field in promoting heat transfer is reduced slightly as the electrode is moved towards the wall. The difference increases with heat flux, corresponding to increased rate of generation of vapour. Further research will be necessary to determine the cause, which might depend simply on obstruction of the bubble motion or the change of the electric field by the presence of bubbles. This may also explain, in part, possible disagreements between research groups. 
Karayiannis and $\mathrm{Xu}$ [19] simulated the effect of the presence of bubbles on the electric field lines and reported distortion in the electric field strength in the vicinity of bubble. The simulation results showed a $30 \%$ increase in the electric field strength on the sides of the bubble while at the bottom of the bubble a slight decrease in the value of $\mathrm{E}$ was reported; maximum value of $\mathrm{E}$ was found at the top of the bubble [19]. Thus the presence of bubbles may be responsible for the nonuniformity of the local electric field which may lead to change in the bubble dynamics and heat transfer enhancement.

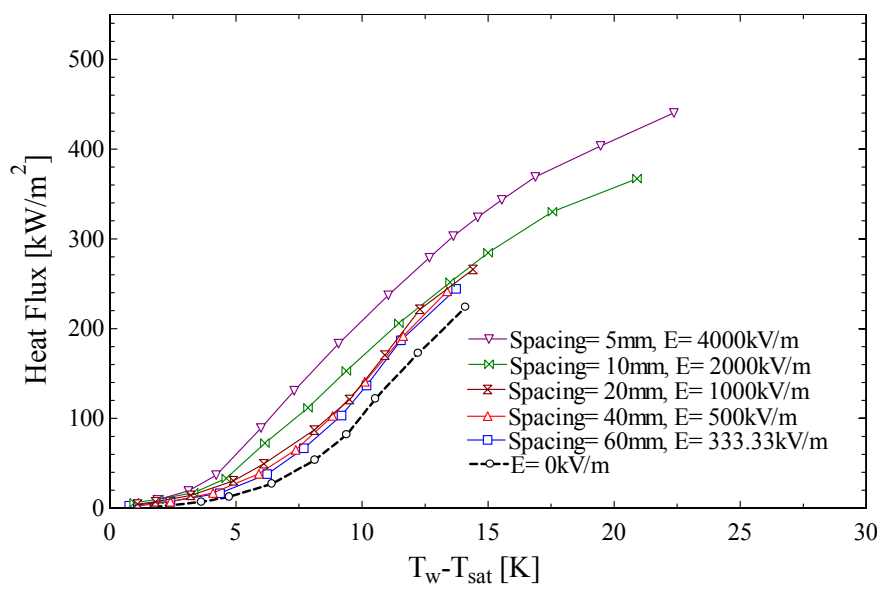

Figure 13 EHD results with variable electrode spacing for an applied voltage of zero and $20 \mathrm{kV}$ and decreasing heat flux.

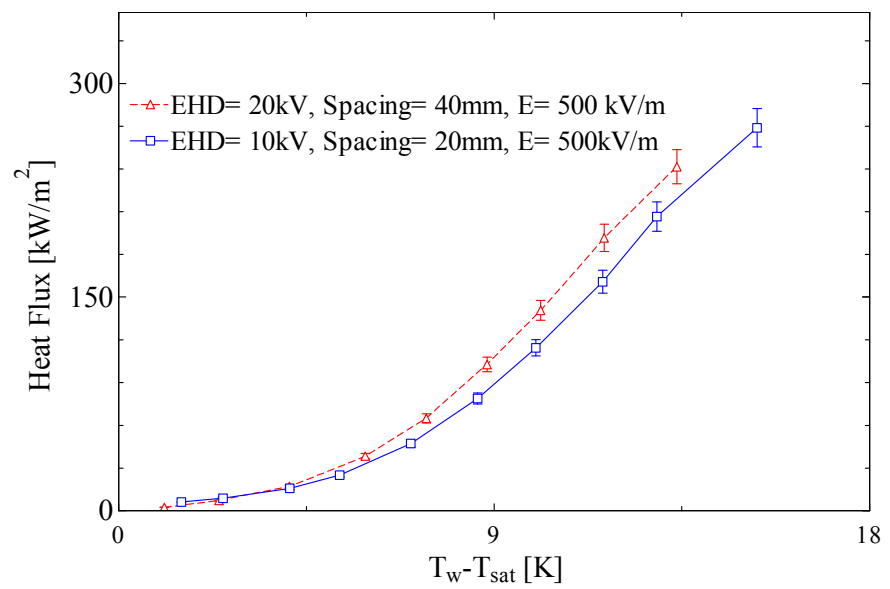

Figure 14 Effect of electrode spacing for the same electric field strength.

\subsection{Compound Enhancement}

The compound enhancement effect of EHD and surface finish is shown in figure 15. The present results for a rough surface are compared with the smoother surfaces of Hristov et al. [18] and Zaghdoudi and Lallemand [21] for electric fields $\mathrm{E}=$ $4000 \mathrm{kV} / \mathrm{m}$ and $\mathrm{E}=1000 \mathrm{kV} / \mathrm{m}$ respectively. As shown previously in figure 8 for $\mathrm{E}=0 \mathrm{kV} / \mathrm{m}$, increasing surface roughness reduces the wall superheat for a given heat flux. The application of an electric field causes a further reduction in wall superheat in all cases, i.e. the compound enhancement of pool boiling - application of EHD and change in the surface roughness- was found to move the boiling curve towards the left as shown in the figure 15.

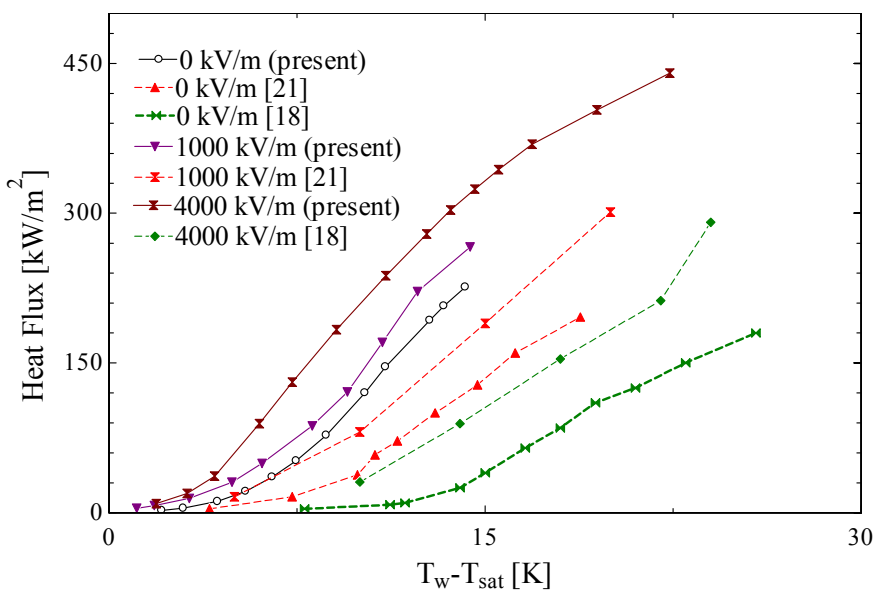

Figure 15 Effect of compound enhancement

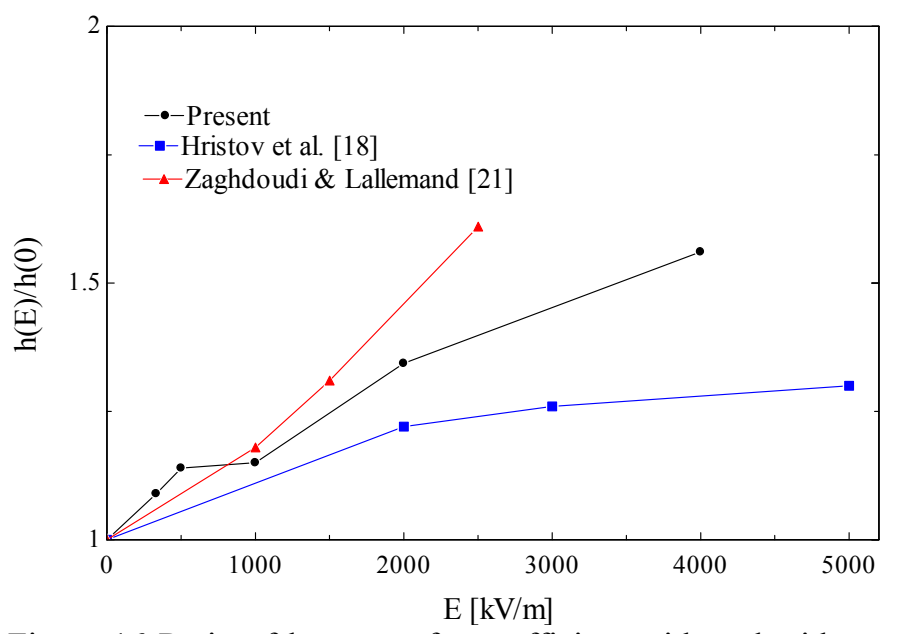

Figure 16 Ratio of heat transfer coefficient with and without EHD at $100 \mathrm{~kW} / \mathrm{m}^{2}$

Figure 16 shows the augmentation ratio of the heat transfer coefficient $h(E) / h(0)$ for each data set at $100 \mathrm{~kW} / \mathrm{m}^{2}$. For fields above $1000 \mathrm{kV} / \mathrm{m}$, the augmentation is highest for the surface believed to have the intermediate value of roughness. The comparison is only an indication because of the missing data for actual surface roughness and differences in the electrode geometry and spacing. The free area of the mesh electrode used by Hristov et al. [18] was 56\%, Zaghdoudi and Lallemands [21] used a similar one at $54 \%$ and the present case was $64 \%$. Zaghdoudi and Lallemand [21] estimated by numerical simulation that the non-uniformity of the electric field increased with electrode spacing. They performed their experiment with a fixed electrode spacing $\mathrm{d}=10 \mathrm{~mm}$ and varied the electric potential from 0 to $25 \mathrm{kV}$; in the present study the electric potential was kept constant at $20 \mathrm{kV}$ while the electrode spacing was changed from $5 \mathrm{~mm}$ to $10,20,40,60$ $\mathrm{mm}$. Further experiments are required with full control of all the variables in order to understand the interaction of surface roughness and electrohydrodynamic enhancement.

\subsection{EHD effect on CHF}

Figure 17 shows the increase in the CHF with the application of the electric field. The CHF at zero electric field was 225 $\mathrm{kW} / \mathrm{m}^{2}$. The CHF increased by a factor of 2.0 at $4000 \mathrm{kV} / \mathrm{m}$. It was observed during the present studies that near the critical heat flux small bubbles from the heating surface tend to coalesce, eventually forming bubble columns. The heating 
surface was covered by these bubble columns, which were observed to swirl in the pool. It is suggested that these bubble columns obstruct bulk liquid from reaching the heating surface, promoting the formation of vapour patches and eventually leading to rapid temperature rise and dryout. Similar bubble behavior was observed by Wang et al. [35] during the pool boiling experiments with liquid nitrogen. They reported that the rise in critical heat flux was by a factor of 1.4 at $4000 \mathrm{kV} / \mathrm{m}$. This increase is lower than the present study, which might be due to the difference in electrical properties of the liquid nitrogen and R123, i.e. the relative electrical permittivities of liquid nitrogen and R123 are 1.42 [35] and 3.42 [21] respectively.

Under the application of electric field, it was observed during the present studies, that the big mushroom like bubble columns were pushed against the heating surface and they appeared to be converted into smaller columns. This suppression may be responsible for changes in the bubble dynamics and more liquid may be available for the heating surface at even higher heat flux values, which may give rise to increase in the CHF. However, the authors feel that this requires further closer observation and examination.

The ratio of the CHF with to that without EHD obtained in the present experiments lies between the results of Hristov et al. [18] and Zaghdoudi and Lallemand [21]. The models in comparison do capture the correct trend with Berghmans [27] highest and Johnson [30] the lowest enhancement ratios.

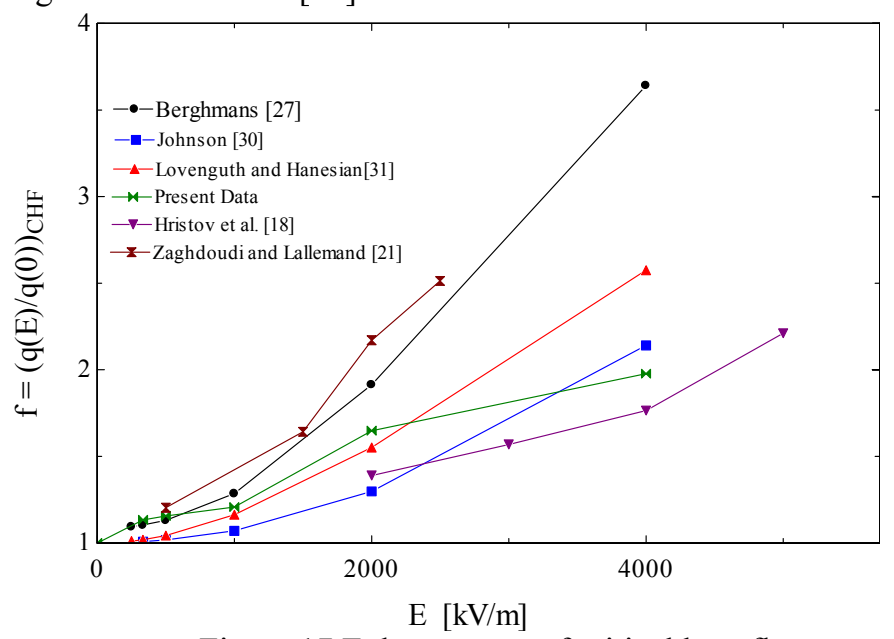

Figure 17 Enhancement of critical heat flux

\subsection{Conclusions}

The paper describes the results of R-123 pool boiling experiments on a sand blasted surface with and without the application of a high intensity electric field.

The following conclusions can be stated:

1. Surface characteristics have an important role to play in nucleate pool boiling and CHF.

2. The application of a sufficiently high electric field can eliminate hysteresis. In the present study with a sand blasted surface this occurred at fields exceeding $1000 \mathrm{kV} / \mathrm{m}$.

3. The application of EHD on such a rough surface can result in significant heat transfer enhancement, especially at low heat flux while at the same time the CHF can increase by a factor of 2.0 at $4000 \mathrm{kV} / \mathrm{m}$

4. The relationship between the applied electric field and the spacing for given field strength may effect the enhancement reported. This may be related to the non - uniformity of the electric field distribution, which increases as the gap increases or to the blockage of the departure vapour stream at high heat flux. This should be examined further.

5. The compound effect of surface roughness (passive technique) and EHD (active technique) may lead to significant enhancement of heat transfer which makes this topis worth further investigation.

EHD enhancement is expected to be higher in a non-uniform electric field and this will be tested next.

\section{Acknowledgements}

This work was supported by Government of Pakistan under a scholarship programme. 


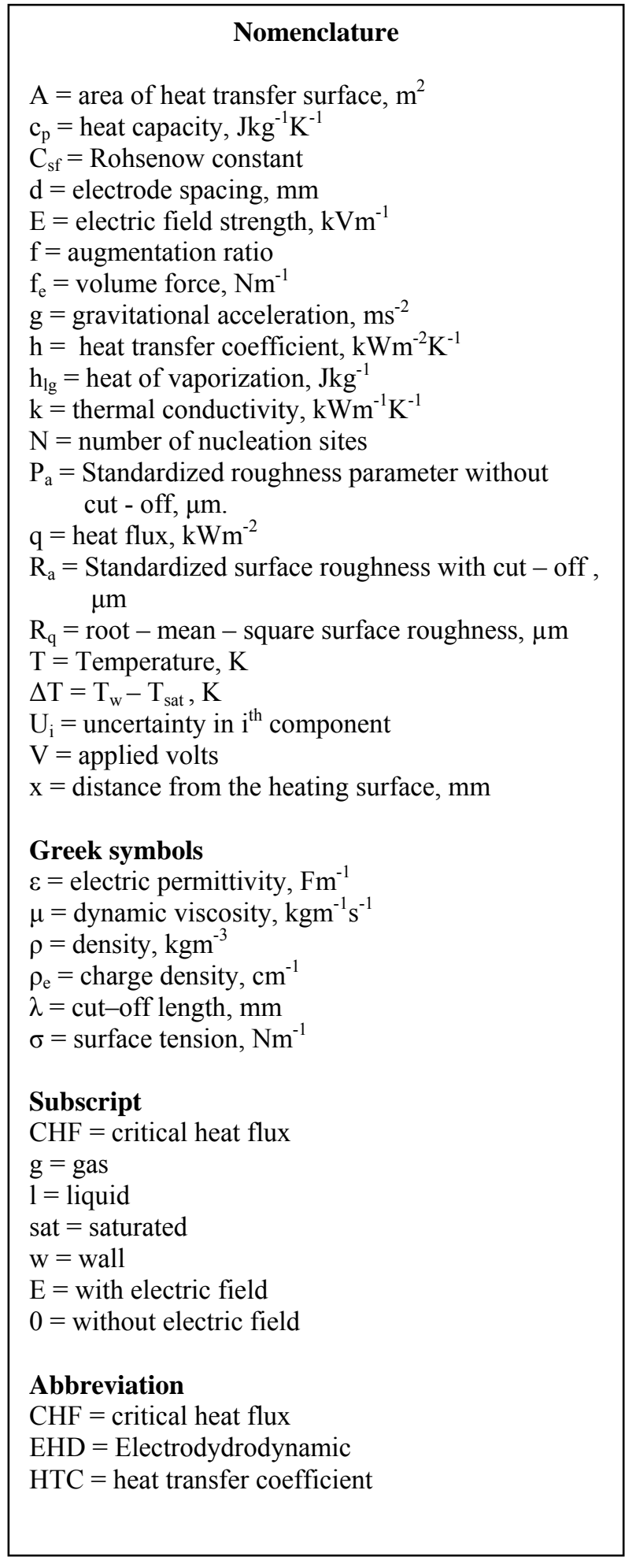

\subsection{References}

1. Reay D.A., "Review Paper Heat transfer enhancement - a review of techniques and their possible impact on energy efficiency in UK", Heat Recovery Sys \& CHP 11(1991) 1-40.

2. Pioro I.L. and Rohsenow W., "Nucleate Pool- boiling heat transfer I: review of parametric effects of boiling surface" International J Heat and Mass Transfer 47 (2004) 5033-5044.
3. Das A.K. and Das P.K., "Nucleate boiling of water from plain and structured surfaces", Experimental Thermal \& Fluid Sciences 31(2007) 967-977.

4. Benjamin R.J. and Balakrishnan A.R., " Nucleation site density in pool boiling of saturated pure liquids : Effect of surface micro roughness and surface and liquid physical properties", Experimental Thermal \& Fluid Sciences 15 (1997) 32-42.

5. Hsieh S.S. and Weng C.J., " Nucleate pool boiling from coated surfaces in saturated R-134(a) and R407(c)" , International J Heat Mass Transfer 40(1997) 519-532.

6. Kang M.G., " Effect of surface roughness on pool boiling heat transfer", International J Heat and Mass Transfer 43 (2000) 4073-4085.

7. Gorenflo D, Chandra U, Kottoff S and Luke A, "Influence of thermophysical properties on pool boiling of refrigerants", International $\mathrm{J}$ of Refrigeration 27 (2004) 492-502.

8. Luke A, "Pool boiling heat transfer from horizontal tubes with different surface roughness", International J of Refrigeration 20 (1997) 561-574.

9. Luke A, Baumhögger and Scheunemann P, “ Multiphase Science and Technology, 12 (2000) 1729.

10. Luke A, "Preparation and analysis of different roughness structures for evaporator tubes", Heat Mass Transfer 45 (2009) 909-917.

11. Luke A, "Preparation, measurement and analysis of microstructure of evaporator surfaces", International $\mathrm{J}$ of Thermal sciences 45 (2006) 237-256.

12. Kottof S, Gorenflo D, Danger E and Luke A, " Heat transfer and bubble formation in pool boiling: effect of basic surface modification for heat transfer enhancement", International J of Thermal Sciences, 45 (2006) 217-236.

13. Ferncic K. and Golobic I., " Surface effects on pool boiling CHF" , Experimental Thermal \& Fluid Sciences 25 (2002) 565-571.

14. Theofanous T.G. and Dinh T.N., "The boiling crisis phenomenon Part II: dryout dynamics and burnout", Experimental Thermal \& Fluid Sciences 26 (2002) 793-810.

15. P.H.G Allen and T.G. Karayiannis, " Review paper Electrohydrodynamics Enhancement of heat transfer and fluid" , Heat Recovery Systems \& CHP, 15 (1995) 389-423.

16. Yabe A., Nakayama W. and Marco P. D., Enhancement techniques in pool boiling, Chapter 5, Hand book of Phase change: Boiling and Condensation edited by Kandlikar S.G., Shoji M. and Dhir V.K. Taylor and Francis 1999.

17. Pohl H.A., "Dielectrophoresis: The behavior of neutral matter in nonuniform electric field", Cambridge University Press (1978).

18. Hristov Y., Zhao D., Kenning D.B.R., Sefiane K., Karayiannis T.G. , "A study of nucleate boiling and critical heat flux with EHD enhancement" , Heat Mass transfer 45 (2009) 999-1017.

19. Karayiannis T.G. and Xu Y., " Electric field effect in boiling heat transfer. Part A: Simulation of electric field and electric forces", Enhanced Heat Transfer 5 (1998) 217-229. 
20. T. Yokoyama, T. Yamazaki, Y. Kubo, Ogata J. and Kawada A., Y. Ooki " The effect of an electric field on boiling heat transfer of fluorocarbon R-11" Proceedings of the XVIII International centre for Heat and Mass Transfer, Dubrobnik, Yugoslavia, 1986, pp 140-151.

21. Zaghdoudi M.C. and Lallemand M., “ Pool boiling heat transfer enhancement by means of high DC electric field", The Arabian J for Science and Engineering 30 (2005) 189-212.

22. Baboi N.F. and Bologa M.K., " Some features of ebullition in Electric field", Applied electrical Phenomenon (USSR) 20 (1968) 57-70.

23. Karayiannis T.G., " EHD boiling heat transfer enhancement of R-123 and R11 on a tube bundle", Applied Thermal Engineering 18 (1998) 809-817.

24. Ohadi MM and Paper RA, "EHD Enhancement of shell-side boiling heat transfer coefficient of R123/oil mixture", ASHRAE Transactions, vol. 90, pt. 2, pp 427-434, 1992.

25. Basu D.K., " Effect of electric field on boiling hysteresis in carbon tetrachloride" , International J Heat and Mass Transfer., 16 (1973) 1322-1324.

26. Cooper P., " EHD Enhancement of Nucleate boiling” , J of Heat Transfer 112 (1990) 458-464.

27. Berghmans J., " Electrostatic fields and the maximum heat flux", Int. J Heat and Mass Transfer 19 (1976) 791-797.

28. Zuber N. , "Hydrodynamics of boiling heat transfer", AEC Report No. AECU-4439, Physics and Mathematics (1959).

29. Markels M. and Durfee R.L., "The effect of applied voltage on boiling heat transfer", AICHE J 10 (1), 106-109 (1964).

30. Johnson R.L., " Effect of an electric field on boiling heat transfer”, AIAA J. 6 (8), 1456-1460 (1968).

31. Lovenguth R.F. and Hanesian D., “ Boiling Heat transfer in the presence of non uniform, direct current electric fields", International Engg. Chem. Fundam 10(1971) 571-576.

32. Coleman W. H. and Steele G. W., "Experimentation and uncertainty analysis for engineers", J Wiley \& sons (1989).

33. Jabardo J.M.S. and Silva E.F.D., "Evaluation of the Rohsenow correlation through experimental pool boiling of Halocarbon refrigerants on cylindrical surfaces", J Braz. Soc. of Mech Sci Eng 24 (2004) 218-230.

34. Shahryari A, Kamal W, Omenovic S, " The effect of surface roughness on the efficiency of the cyclic potentiodynamic passivation (CPP) method in the improvement of general and pitting corrosion resistance of 316 LVM stainless steel" , Material Letter 62 (2008) 3906-3909.

35. Wang P., Lewin P.L., Swaffield D.J. and Chen G., “ Electric field effects on boiling heat transfer of liquid nitrogen", Cryogenics 49 (2009) 379-389. 